\title{
Improving Intrusion Detection by the Automated Generation of Detection Rules
}

\author{
Abdulrazaq Almutairi \\ School of Electronic, Electrical and Systems \\ Engineering \\ Loughborough University \\ Leicestershire, UK
}

\begin{abstract}
Rule Based Detection Systems have been successful in preventing attacks on network resources, but suffer a problem in that they are not adaptable in cases where new attacks are made i.e. they need human intervention for investigating new attacks. This paper proposes the creation of a predictive intrusion detection model that is based on usage of classification techniques such as decision tree, Nä̈ve Bayes, neural network, and fuzzy logic to generate new rules. The proposed model in this paper consists of two stages. The first stage uses either a Decision tree (J48 based on C4.5) or Nä̈ve Bayes classifier based on the results obtained in experiments while the second stage is based on a hybrid module that uses both a neural network (MLP) and fuzzy logic. Training and evaluation phases used randomly selected connections in a subset of the KDD'99 intrusion detection data set. A selected set of features has been extracted from those connections using a subset evaluation algorithm. This paper shows how the proposed system has been trained detailing parameters that affect the training process; it also details results obtained in the evaluation process including detection and false positive rates.
\end{abstract}

\section{Introduction}

In the context of information technology, intrusion can be defined as a series of attempts in order to compromise the security of a (often network based) resource [1]. Network based systems or resources require constant monitoring in order to ensure that malicious activities can be contained [2]. An Intrusion Detection System (IDS) is responsible for monitoring network traffic and based on a set of rules that raise alerts for information security officers when malicious traffic is detected [2].

Although IDSs are successful in terms of preventing attacks on network resources, they are not adaptable in cases where new attack attempts are made i.e. they need human intervention for investigating new attacks [3]. Furthermore, an IDS could become a bottleneck where it is employed on a busy network. An IDS require time for processing network data before it can be released to the production network [3].

\author{
David Parish \\ School of Electronic, Electrical and Systems \\ Engineering \\ Loughborough University \\ Leicestershire, $U K$
}

A solution to address the above problem is to create a system that is based on Machine Learning. This signaturebased system will use existing IDS such as Snort to compare packet signatures with rules defined by Snort and the packet suspected to be malicious are subjected to be passed to an intelligent model that has been trained to detect malicious contents [3] [4]. Hence, Snort will act as the first level of filtering reducing the amount of traffic that becomes a candidate for further investigation using the intelligent model. Overall, this reduces human intervention as the intelligent trained model is responsible for deciding if certain sets of packets are malicious or otherwise. If a set of packets are found to be malicious an automated signature will be created that will update a set of rules used by Snort.

The novelty is offered by integration of a training model for detecting misuse in the incoming network data packets with a reasoning model that is applied on the outliers (uncategorised data packets) [4]. The result of reasoning model is in the form of a rule that can be used by IDS actively on a production level system to filter malicious data packets.

Section 2 provides the aim of this paper. Section 3 discusses related research conducted in this domain. Section 4 provides a brief background with regards to classifier algorithms viz. Naïve Bayes, Decision Tree, Neural Network, and Fuzzy Logic. In Section 5, a detailed description of our research methodology has been provided. Section 6 provides a description of KDD'99 intrusion detection data set and Section 7 discusses the metrics used for attribute selection in the KDD'99 intrusion detection data set. Section 8 describes the experiments conducted and discusses results obtained after passing the data through Naïve Bayes and Decision Tree algorithms respectively. Section 9 discusses the reasoning mechanism and how it was implemented using a neural network and fuzzy logic. Section 10 provides the conclusion of this paper and finally, Section 11 provides a description of future work to be conducted.

\section{Aim}

The aim of this paper is to provide a comparative study of classification algorithms for the purpose of creating a training model and a reasoning mechanism used for 
misuse detection. In this comparative study we present results in terms of a confusion matrix and metrics such as true-positive, false positive, true negatives and false negatives. Also presented are comparisons between expected and predicted classes of KDD'99 intrusion detection data [5].

\section{Related Research Work}

One of the most well-known works in the context of using data mining for intrusion detection is by Stefan Axelsson [6]. In this the model created uses the maximised posterior probabilities as parameters provided by Bayesian algorithm [6]. As a result the false alarm rate that is usually shown by IDSs is reduced. In our research the aim has been to create new signatures based on reasoning of outlier instances. In other words, our research is complementing research work by provision of a reasoning module [6].

Intrusion Detection using Data Mining uses a real-time network intrusion detection system for detection of misuse [7]. It employs association rules; characteristic rules and meta rules to provide results with regard to deviation from normal network activity.

Similar to that is Mining Audit Data for Automated Models for Intrusion Detection. W. Lee outlined a datamining framework for constructing intrusion detection models [8]. The key idea is to first apply data mining programs to audit data to compute frequent patterns, extract features, and then use classification algorithms to compute detection models.

In Chang et al. the method of back propagation by sample query and attribute query has been used for intrusion detection to identify and analyse feature of training data. The main contribution of Chang has been a reduction in processing time and storage of data instances [9].

Perhaps, the closest research work that has been conducted is by Barbara, where a training system to classify, unknown and false alarm instances was built [10]. Furthermore they used and analysed the unknown instance by following their audit trail in order to provide concrete result informing if the data instance is an outlier. Our research goes a step further by creating a rule signature where by IDSs rule holder can be updated automatically.

\section{Brief Background}

For misuse detection, we have used four classifiers viz. Naïve Bayes, Decision Tree, Neural network, and fuzzy logic. This section provides a brief description of each technique. For detailed background, see [11] [12] [13] [14] [15].

\subsection{Naïve Bayes}

Bayesian reasoning is applied to decision-making that deals with probabilistic inference i.e. the knowledge about previous events is used to predict future events [12]. In a Naïve Bayes classifier the availability or unavailability of certain attributes is not related to the availability or unavailability of another attribute. Naïve Bayes provides an advantage when making decisions based on small amounts of training data to compute mean and variance of the attributes to compute its class. Bayes theorem provides a method of calculating the posterior probability, $\mathrm{P}(\mathrm{c} \mid \mathrm{x})$, from $\mathrm{P}(\mathrm{c}), \mathrm{P}(\mathrm{x})$, and $\mathrm{P}(\mathrm{x} \mid \mathrm{c})$. The Naive Bayes classifier assumes that the effect of the value of a predictor ( $\mathrm{x}$ ) on a given class (c) is independent of the values of other predictors [14]. This offers conditional independence. Bayes algorithm is explained by following:

$$
\begin{aligned}
& P(c \mid x)=\frac{P(x \mid c) P(c)}{P(x)} \\
& P(c \mid X)=P\left(x_{1} \mid c\right) * P\left(x_{2} \mid c\right)^{*} \ldots P\left(x_{n} \mid c\right) * P(c)
\end{aligned}
$$

where,

- $\quad \mathrm{P}(\mathrm{c} \mid \mathrm{x})$ is the posterior probability of class given attribute.

- $\quad \mathrm{P}(\mathrm{c})$ is the prior probability of class.

- $\mathrm{P}(\mathrm{x} \mid \mathrm{c})$ is the likelihood which is the probability of attribute given class.

- $\mathrm{P}(\mathrm{x})$ is the prior probability of attribute.

\subsection{Decision Tree}

A decision tree classifies a data set through a sequence of decisions where a decision on a current node facilitates a decision to be made on the following nodes [11]. This forms an n-array tree structure where a decision is made by traversing from a root node to a leaf node where the leaf node represents a class. Nodes are formed of attributes or features from the data set. One of the best-known decision tree algorithms is J48 - an open source implementation of the $\mathrm{C} 4.5$ algorithm for decision trees, available through Weka [11] [13]. J48 offers the handling of a variety of input data types for instance nominal, textual and numeric and is high in performance. The algorithm operates as follows:

- The algorithm operates over set of instances used for training, $C$.

- If all instances in $C$ are in class $P$

- Then create a node $P$ and end.

- Else select attribute $F$ and create division node.

- Partition the instance $C$ into subset of values $\left(V_{1 . . n}\right)$ for attribute $F$.

- Apply the algorithm recursively to each of the subsets of instance $C$. 


\subsection{Neural Network}

An Artificial Neural Network is a computational model inspired by the principles of an animal's central nervous system. This model has the capabilities of machine learning as well as pattern recognition. It has been described as a system that adapts its structure in the learning phase; this adoption is based on external or internal information flowing through the system [14].

The most commonly used architecture of supervised neural networks is Multi-Level Perceptron (MLP). That architecture contains a number of layers (one input layer, a number of hidden layers, and one output layer), each layer contains a number of processing units called neurons. Each neuron is connected with a weight to a neuron in the following layer. MLP uses the back propagation algorithm in the training process. In that algorithm, the input data is passed to the neural network. Then, the output of the network is compared to the desired output to compute the error. That error is used to adjust the weights in order to get closer to the desired output [14]. The error calculation and weight changes are explained by following [14]:

$$
e_{j}(n)=d_{j}(n)-y_{j}(n)
$$

$$
\varepsilon(n)=\frac{1}{2} \sum_{j} e_{j}^{2}(n)
$$

Where,

- $e_{j}$ is the calculated error for neuron $\mathrm{j}$.

- $\mathrm{n}$ is the index of training data.

- $d_{j}$ is the desired value.

- $y_{j}$ is the produced value by neuron $\mathrm{j}$.

- $\varepsilon$ is the error of entire output.

- $w_{j i}$ is the weight of the connection between neuron $\mathrm{i}$ in a layer and neuron $\mathrm{j}$ in the following layer.

- $\quad \mu$ is the learning rate (a value between 0.2 and $0.8)$.

\subsection{Fuzzy Logic}

Fuzzy logic is a computational approach based on human language rules. The fuzzy system translates the defined rules to mathematical equivalent [15]. A system shown in Fig.1 consist of a fuzzifier, interference engine, rules base, and defuzzifier. Fuzzy systems work as follow [15]:

- $\quad$ The fuzzifier converts the crisp inputs to a fuzzy set by using specified membership functions for each input.
- Based on the defined rules, the interference engine produces a fuzzy output.

- $\quad$ The fuzzy output is converted to a crisp value using the membership functions defined for defuzzification.

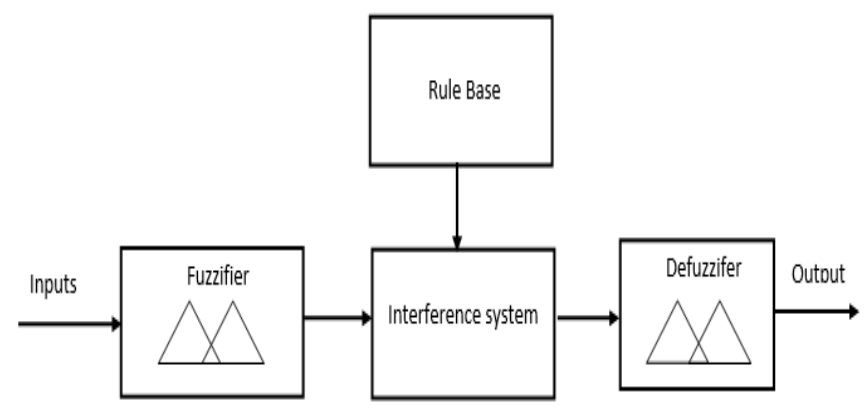

Figure 1. Fuzzy logic Components

\section{Research Methodology}

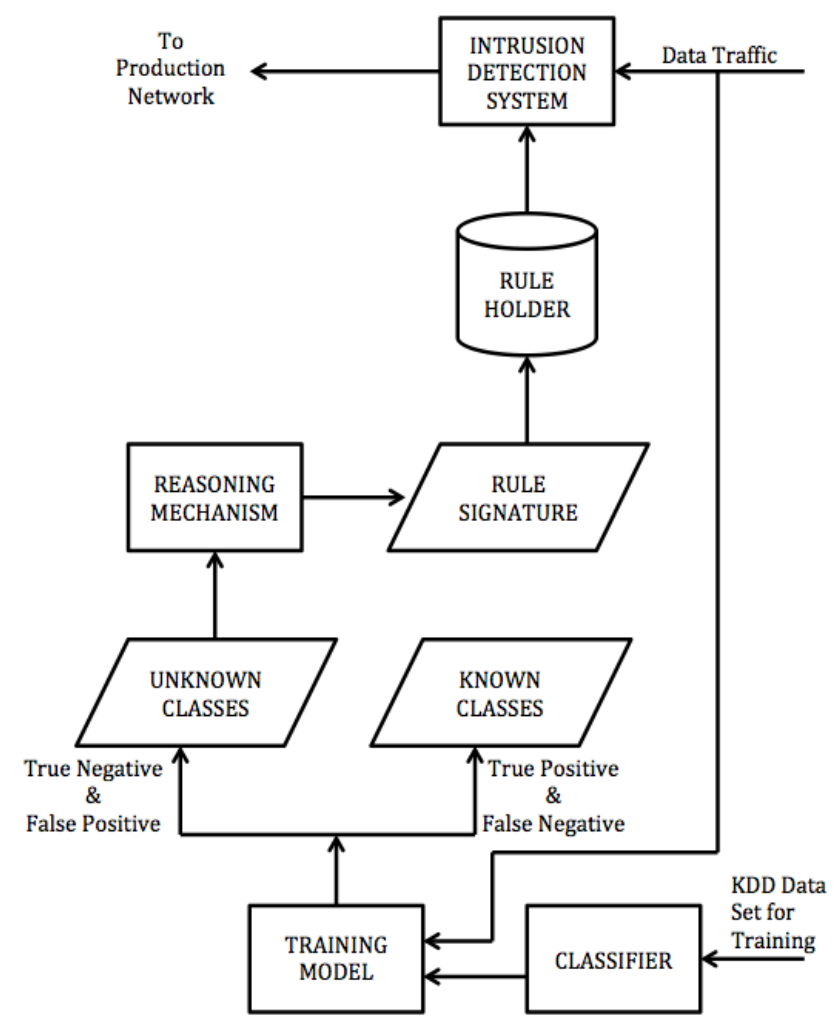

Figure 2. High Level View of Research Process

The research process consists of the following elements (see Fig. 1): 
- Data Set and Categorisation: The first step in the research process is to find a reliable high quality network traffic data set where each packet has been labelled so that the training model is created as a result the classification can be used reliably.

- Feature Selection: The network packets in the data set are then passed through an attribute evaluator in order to extract a set of features that can be used effectively to detect intrusions. Unessential features are known to be not only a bottleneck in terms of cost of computation but also are factors that contribute towards increased error rates [16].

- Classifier Model Creation: The purpose of this step is to use a classification algorithm using Decision Tree and Naïve Bayes that can compute a model that uses the most discriminating features in an instance of a data packet to describe a class (concept) [12]. This is done by training a classifier using a pruned set of features where the objective is that the model created are more generic than the rules (as compared to Snort) and hence, it outperforms this in accuracy and effectiveness when compared to general rule based signature matching systems.

- Reasoning Mechanism Creation: The purpose of this step is to provide another stage for classifying the network traffic if the first stage fails to classify it. The reasoning mechanism is based on a hybrid model built using neural network (MLP) and fuzzy logic.

\section{Description Of Data Set}

The data set used in this paper is the KDD'99 intrusion detection data set. This data set is based on a 1998 DARPA initiative and has been used by researchers for evaluation of various intrusion detection methodologies in past. The data is collected as a result of a setup of a fictitious military network with a number of target machines running various services. A sniffer was used to record all network data using a raw TCP/IP dumps [5].

The data set consists of 41 discrete and continuous attributes and has 22 attack classes and 1 normal class, where each instance in data set has been categorised as one class.

The attacks are further divided into 4 categories:

- Denial of Service Attack Category (DoS) where a target host is compromised by request for service from multitude of machines. (e.g. syn flood)

- User to Root Attack Category (U2R) where an attacker attempts to get unauthorised access to the root level of a target system. (e.g. buffer overflow attacks)

- Remote to User Attack Category (R2L) where a hacker tries to take control of a remote machine by exploiting vulnerabilities of the system. (e.g. guessing passwords)

- Probing Attack Category (probe) where an attacker scans the machines (generally on network) in order to collect useful information (for instance, services running) about those machines. (e.g. port scanning)

\section{Selection Of Important Features}

For selection of important attributes in the network data set, Correlation-based Feature Selection (CFS) was employed. A search algorithm as well as a classifier function is used by CFS to evaluate the importance of each feature and provide a subset of features [17]. The heuristic that is used by CFS indicates that important features are highly correlated to the class; however, they are uncorrelated from each other [17]. In a data-mining context, this approach is based on information gain that measures the importance of each attribute for predicting the class based on the calculated entropy of that attribute. An attribute with entropy value approaching 0 will have information gain approaching 1 [18].

$$
H[D]=-\sum_{j=1}^{|C|} P\left(c_{j}\right) \log _{2} P\left(c_{j}\right)
$$

Where $\mathrm{C}$ is the desired class.

Information gained by removal of an attribute can be computed as a difference of entropy before removal to entropy after removal of that attribute [13].

$$
\operatorname{gain}\left(D, A_{i}\right)=H[D]-H_{A_{i}}[D]
$$

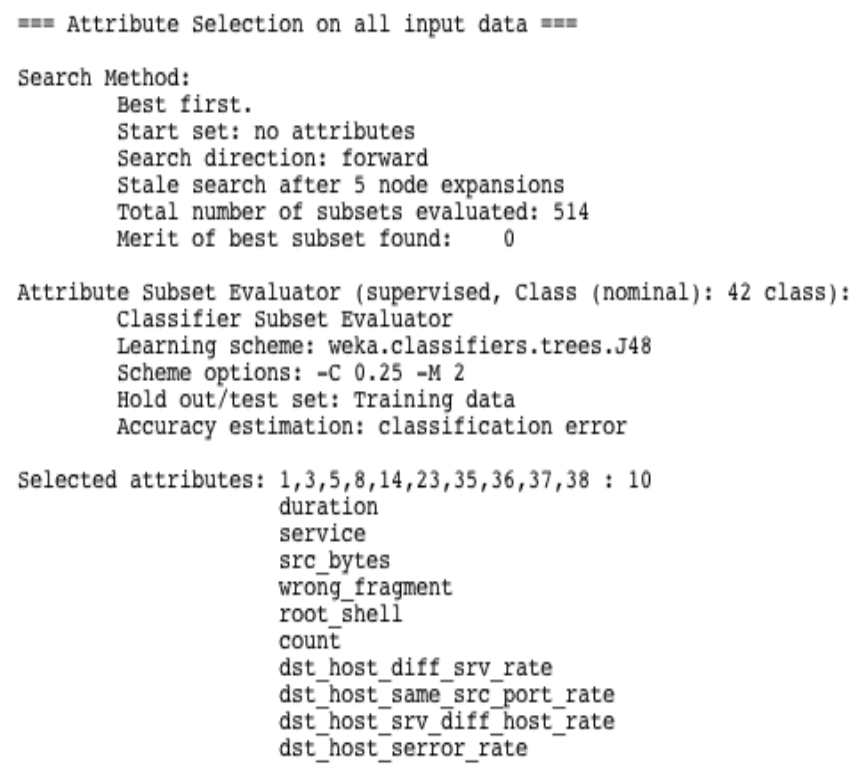

Figure 3. Attributes Selected Upon Using CFS Evaluator and Depth First Search 


\section{First Stage of Classification}

For this paper, $10 \%$ of the whole KDD'99 intrusion detection data set was used. This data set represents a concise version of the whole data. This data set contained approximately half a million classified instances of network data packets [5]. For the purpose of testing the effectiveness of the created model, $34 \%$ of this data set approximates to 168,000 known classified instances were used. A training model has been created upon considering two model creation strategies:

1. All Classes: In this case, a training model has been created upon considering all the classes described in the KDD intrusion detection data set.

2. Two Classes: In this case, a training model has been created by categorising the KDD data set into only two different classes; normal and malicious.

\subsection{All Classes Based Model Creation Strategy}

Table 1 shows the results of instances classified correctly and incorrectly upon use of Naïve Bayes and Decision Tree (J48) algorithms for the creation of the training model using the all classes' model creation strategy. It is noted that the results of correctly classified instances by employing Decision Tree are slightly better than Naïve Bayes [11] [12].

Table 1. Results For Naïve Bayes And Decision Tree Using All Classes Model Creation Strategy

\begin{tabular}{|c|c|c|}
\hline $\begin{array}{c}\text { Instances } \\
\text { Classified }\end{array}$ & Naïve Bayes & Decision Tree \\
\hline Correctly & $91.82 \%(154228)$ & $99.95 \%(167890)$ \\
\hline Incorrectly & $8.18 \%(13739)$ & $0.04 \%(77)$ \\
\hline
\end{tabular}

Table 2. Accuracy / Class For Naïve Bayes And Decision Tree Using All Classes Model Creation Strategy

\begin{tabular}{|c|c|c|c|c|}
\hline \multirow{2}{*}{ Class } & \multicolumn{2}{|c|}{ True Positive } & \multicolumn{2}{c|}{ False Positive } \\
\cline { 2 - 5 } & $\begin{array}{l}\text { Nä̈ve } \\
\text { Bayes }\end{array}$ & $\begin{array}{l}\text { Decision } \\
\text { Tree }\end{array}$ & $\begin{array}{l}\text { Nä̈ve } \\
\text { Bayes }\end{array}$ & $\begin{array}{c}\text { Decision } \\
\text { Tree }\end{array}$ \\
\hline Normal & 0.617 & 0.999 & 0 & 0 \\
\hline Buffer Overflow & 0.462 & 0.615 & 0.001 & 0 \\
\hline Load Module & 0.4 & 0.2 & 0.001 & 0 \\
\hline Perl & 0 & 0 & 0 & 0 \\
\hline Neptune & 0.999 & 1 & 0.001 & 0 \\
\hline Smurf & 0.998 & 1 & 0 & 0 \\
\hline Guess Passwd & 0.952 & 1 & 0.025 & 0 \\
\hline Pod & 0.987 & 1 & 0 & 0 \\
\hline Teardrop & 0.988 & 0.997 & 0 & 0 \\
\hline Portsweep & 0.111 & 0.979 & 0.01 & 0 \\
\hline IPsweep & 0.97 & 0.993 & 0.007 & 0 \\
\hline
\end{tabular}

\begin{tabular}{|c|c|c|c|c|}
\hline Land & 0.75 & 1 & 0 & 0 \\
\hline FTP Write & 0 & 0.5 & 0.002 & 0 \\
\hline Back & 0.984 & 0.996 & 0 & 0 \\
\hline IMAP & 1 & 0.4 & 0 & 0 \\
\hline Satan & 0.894 & 0.986 & 0.002 & 0 \\
\hline PHF & 1 & 0 & 0.011 & 0 \\
\hline NMap & 0.457 & 0.988 & 0.001 & 0 \\
\hline Multihop & 0 & 0 & 0.006 & 0 \\
\hline Warezmaster & 0.75 & 1 & 0.002 & 0 \\
\hline Warezclient & 0.107 & 0.979 & 0 & 0 \\
\hline Spy & 0 & 0 & 0 & 0 \\
\hline Rootkit & 0.667 & 0 & 0.012 & 0 \\
\hline
\end{tabular}

Table 2 shows the consolidated comparative results per class for each classifier for metrics True-Positive (TP) and False-Positive (FP). It is observed that although Naïve Bayes has a high TP; it is skewed by the results of FP. On the other hand, Decision Tree has almost a high consistent TP and consistent low FP.

The results also show that high FP rate has been observed in classes corresponding to probing and remote to local attack categories. This indicates the attacks were mainly in categories where the attacker was scanning a machine to understand more about vulnerabilities of network resources and furthermore that these could be initiated from a remote location where a local machine could have been compromised.

\subsection{Two Classes Based Model Creation Strategy}

Table 3 shows the results of instances classified correctly and incorrectly upon use of Naïve Bayes and Decision Tree (J48) algorithms for creation of a training model using the two classes model creation strategy. It is observed that using the training model created by use of Decision Tree is better than Naïve Bayes.

It is further observed that in contrast to the all classes based model creation strategy; Naïve Bayes has performed better in correctly classifying the instances as the results were boosted from $91.82 \%$ to $98.44 \%$.

Table 3. Results for naïve bayes and decision tree using two classes model creation strategy

\begin{tabular}{|c|c|c|}
\hline Instances Classified & Naïve Bayes & Decision Tree \\
\hline Correctly & $\begin{array}{c}98.44 \% \\
(165349)\end{array}$ & $99.96 \%(167898)$ \\
\hline Incorrectly & $1.56 \%(2618)$ & $0.04 \%(69)$ \\
\hline
\end{tabular}

Table 4 presents the combined comparative results per class for each classifier for metrics TP and FP for two classes based model creation strategy. It is observed that Decision Tree consistently has a high true positive rate and consequently a low false positive rate.

Overall, it has been observed that Decision Tree algorithm is more effective in prediction of classes for data instances. Furthermore, the true positive rate of the Decision Tree algorithm is higher in the two classes based 
model creation strategy making this strategy a better choice for the model.

However, it should be noted that the objective of our model is to provide a prediction of data instances with high granularity of categorised class so that any categorised instances can be subjected to further critique using a reasoning mechanism. Because of the aforementioned requirement, we have selected the all classes based training model creation strategy for prediction of classes of data instances.

\section{Table 4.Accuracy / Class For Naïve Bayes And Decision Tree Two Classes Model Creation Strategy}

\begin{tabular}{|c|c|c|c|c|}
\hline \multirow{2}{*}{ Class } & \multicolumn{2}{|c|}{ True Positive } & \multicolumn{2}{c|}{ False Positive } \\
\cline { 2 - 5 } & $\begin{array}{c}\text { Nä̈ve } \\
\text { Bayes }\end{array}$ & $\begin{array}{c}\text { Decision } \\
\text { Tree }\end{array}$ & $\begin{array}{c}\text { Nä̈ve } \\
\text { Bayes }\end{array}$ & $\begin{array}{c}\text { Decision } \\
\text { Tree }\end{array}$ \\
\hline Normal & 0.989 & 0.999 & 0.017 & 0 \\
\hline Malicious & 0.983 & 1 & 0.011 & 0.001 \\
\hline
\end{tabular}

\section{Second Stage of Classification}

The second stage of classification is achieved through a reasoning mechanism. The proposed reasoning mechanism in this paper classifies the network traffic into normal (1) or attack (0). The mechanism is based on a hybrid model consisting of two modules; the first one is based on a neural network while the second one is based on fuzzy logic. Fig.4 gives an overview of the proposed system. The proposed system will classify network traffic as normal if both modules classify it as normal while it will classify it as attack if either of the modules classifies the traffic as attack. Both modules of the hybrid model have been implemented using PHP and MYSQL rather than using Weka due to a known memory issue with Weka. In addition, developing the model using a programming language rather than using a ready-made tool has given more flexibility to customize the model in the way the authors need in their experiments.

The Neural network has the advantage of the ability to work with incomplete and inprecise data. This merit can be employed in an IDS context for detecting attack patterns presented during the training phase but modified by an attacker in order to pass through the system [19]. The flexibility of fuzzy logic can be employed in case of uncertain problems of intrusion detection and allows much greater complexity for IDS [20].

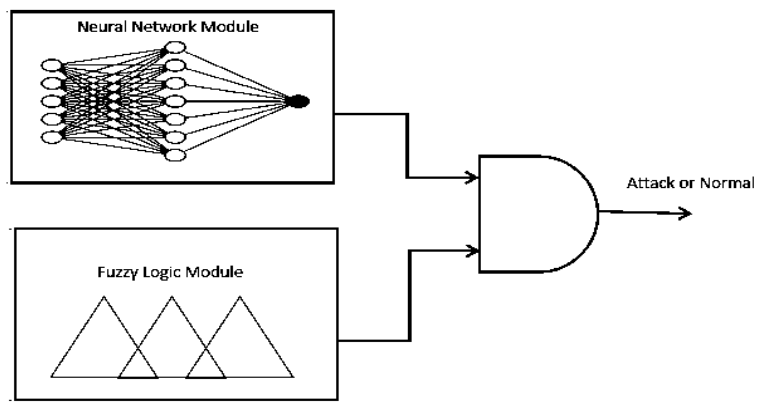

Figure 4. Hybrid Model Overview

The benefit of using the hybrid approach is to increase the intrusion detection rate; because some of attacks may not be detected by one of the modules but the other one may be able to detect them. In other words, one module will overcome some of the module shortcomings of the other in detecting malicious traffic. However, there is a chance of increasing the false positive rate for normal traffic.

The architecture used in the neural network module is MLP. This architecture has three layers. The first layer contains 10 neurons; the hidden layer contains 8 neurons, and one neuron in the output layers. The neural network module was trained using $10 \%$ of the whole KDD'99 intrusion detection data set. It has been trained by setting the max mean square error to .01, and max number of epochs to 3000 . The training data was pre-processed as follows before starting the training process:

1. Feature labelling: Label non-numeric attributes with numeric values. Some features are not represented by a numeric value (e.g. service), whilst numeric values are required by the neural network. For example, each service has been given a number; 1 for telnet, 2 for ftp_data [14].

2. Feature normalization: It has been found that each feature has a different range. Thus, all attributes have been normalized in a way that has made each attribute to have the same range (between 0 and 1). This step helps in making the selected attributes comparable.

3. Remove redundant data: Removing redundant or repeated data from the training data set prevents the training algorithm from being biased in the direction of more frequent records and ignoring less frequent records. The number of the training data set records after removing the duplicates is 142,000 .

On the other hand, the rules of the fuzzy module were created using 10\% of the whole KDD'99 intrusion detection data set as follow:

1. All selected feature apart from 'service' (as it is a discrete value not continuous) have been normalized in a way that has made each attribute to have the same range (between 0 and 1). This 
step helps in simplifying the rules generation process.

2. Three values V1, V2, V3 were selected, where:

$$
\mathrm{V} 1=.043, \mathrm{~V} 2=0.375, \mathrm{~V} 3=.75
$$

3. During the iteration through the training data, each selected feature (apart from 'service') was translated from a numeric value to a description as follow:

$0 \leq$ attribute value $<$ V1 $\rightarrow$ Very Low $(\mathrm{VL})$

$\mathrm{V} 1 \leq$ attribute value $<\mathrm{V} 2 \rightarrow$ Low $(\mathrm{L})$

$\mathrm{V} 2 \leq$ attribute value $<\mathrm{V} 3 \rightarrow \operatorname{High}(\mathrm{H})$

$\mathrm{V} 3 \leq$ attribute value $\leq 1 \rightarrow$ Very High $(\mathrm{VH})$

while the output is either described as normal or attack. The rule was then created in the following form:

If (feature1 is feature1_desc AND feature2 is feature 2 desc AND ...feature10 is feature10_desc) Then output is output_desc

4. The created rule in the previous step would not be added to the rule base, if it was added before to avoid having duplicate rules. The total number of rules added to the rule base is 1343 .

Different values had been tried for V1, V2, V3 but the value selected above gave the best results in terms of false positive and detection rates. The last step in implementing the fuzzy module was the membership functions selection for both inputs (selected feature) and output. Fig.5 shows the membership functions for all inputs apart from the 'service' feature which was handled by a singleton membership function for each value as it is a discrete attribute. The output has two membership functions as shown in Fig.6.

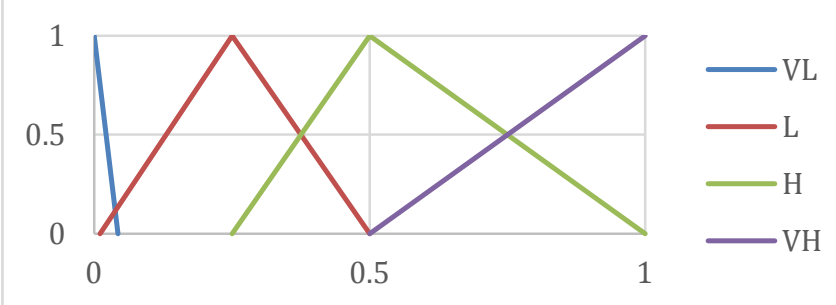

Figure 5. Membership function for the selected feature (not including the 'service' feature)

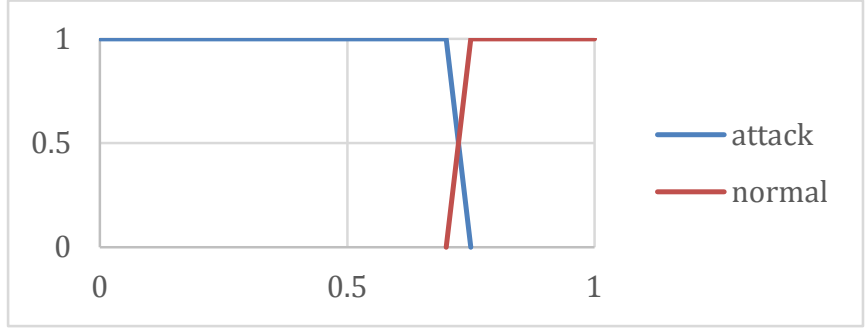

\section{Figure 6. Membership function for the output}

The results of evaluation obtained from the hybrid model after completing the neural network training and the rules generation and membership functions selection for the fuzzy module are shown in table 5. The evaluation process was carried out using $10 \%$ of the whole KDD'99 intrusion detection data set, this data set is different from the data set presented in the neural network training and rules generation stage.

Table 5 shows that the hybrid model achieved a higher detection rate for malicious traffic than both the neural network and fuzzy logic modules individually. On the other hand, the false positive rate of the hybrid model is higher than both the neural network and fuzzy logic each one individually.

Table 5. Results of the hybrid model using Neural Network and fuzzy Logic

\begin{tabular}{|c|c|c|c|c|c|c|}
\hline \multirow{2}{*}{ Class } & \multicolumn{3}{|c|}{ True Positive } & \multicolumn{3}{c|}{ False Positive } \\
\cline { 2 - 7 } & $\begin{array}{c}\text { Neural } \\
\text { Networ } \\
\boldsymbol{k}\end{array}$ & $\begin{array}{c}\text { Fuzzy } \\
\text { Logic }\end{array}$ & $\begin{array}{c}\text { Hybri } \\
\boldsymbol{d} \\
\text { Model }\end{array}$ & $\begin{array}{c}\text { Neural } \\
\text { Networ } \\
\boldsymbol{k}\end{array}$ & $\begin{array}{c}\text { Fuzzy } \\
\text { Logic }\end{array}$ & $\begin{array}{c}\text { Hybri } \\
\boldsymbol{d} \\
\text { Model }\end{array}$ \\
\hline Normal & 0.971 & $\begin{array}{c}0.97 \\
8\end{array}$ & .952 & 0.029 & .022 & .048 \\
\hline $\begin{array}{c}\text { Maliciou } \\
\text { s }\end{array}$ & 0.966 & $\begin{array}{c}.999 \\
5\end{array}$ & .9997 & 0.034 & $\begin{array}{c}0.000 \\
5\end{array}$ & .0003 \\
\hline
\end{tabular}

\section{Conclusion}

Snort monitors network traffic and uses content searching and matching to detect attacks. One of problems with using Snort is the fact that it is not adaptable to detect new attacks. In addition, it generates false alarms at a high rate.

Our experiments can be used to conclude that data mining can be implemented as an added portion to preexisting IDS. When implemented properly, data mining can improve the classification process resulting in a lowered number of false positive alerts. The first stage of our proposed model built using the Decision Tree approach is able to classify most data correctly, has better accuracy rate, detection rate and lower false positive rates. In this paper, we have compared two different training model creation strategies using Naïve Bayes and Decision Tree algorithms. It is concluded that an all classes based training model creation using the decision tree is the most effective as it not only provides a better true positive rate but also a high level in granularity for classification of data 
instances and this has high precedence over a slightly better training model created using two classes model creation strategy.

The second stage of the proposed model (reasoning mechanism) was built using a hybrid approach. The hybrid approach in this paper uses both neural network and fuzzy logic. The benefit of using the hybrid approach is increasing the intrusion detection rate, some of attacks may not be detected by one of the modules but the other one may be able to detect them. The results obtained by this approach achieves a higher detection rate than both the neural network and fuzzy logic each one individually. However, it has a higher false positive rate.

\section{Future Work}

For the next phase of research work, the authors are working on decreasing the false positive rate of the reasoning mechanism by trying a different neural network structure, and different membership functions in the fuzzy module. The authors will also consider developing the proposed model to handle multi-stage attacks by analysing and modelling different multi-stage attacks scenarios.

\section{References}

[1] H.-J. Liao, et al., "Intrusion detection system: A comprehensive review," Journal of Network and Computer Applications, 2013, vol.36, n.1, pp. 16-24.

[2] D.-K. Kang, D. Fuller, and V. Honavar, "Learning classifiers for misuse and anomaly detection using a bag of system calls representation," in Proceedings from the Sixth Annual IEEE SMC Information Assurance Workshop (IAW'05), 2005, pp. 118-125.

[3] M. Roesch, "Snort: Lightweight Intrusion Detection for Networks," in LISA. 1999, vol.99, pp. 229-238.

[4] G. Kim, S. Lee, and S. Kim, "A novel hybrid intrusion detection method integrating anomaly detection with misuse detection," Expert Systems with Applications, 2014. vol.41, n.4, pp. 1690-1700.

[5] KDD'99. KDD Cup 1999 Data. 1999. Available from: https://kdd.ics.uci.edu/databases/kddcup99/kddcup99.html (Access Date: 17 July, 2014).

[6] S. Axelsson, "The base-rate fallacy and its implications for the difficulty of intrusion detection," in Proceedings of the 6th ACM Conference on Computer and Communications Security. 1999, pp.1-7.

[7] T. Abraham, "IDDM: Intrusion detection using data mining techniques," 2001.

[8] W. Lee, and S.J. Stolfo, "A framework for constructing features and models for intrusion detection systems," ACM transactions on Information and system security (TiSSEC), 2000, vol.3, n.4, pp. 227-261.
[9] R.-I. Chang, et al., "Intrusion detection by backpropagation neural networks with sample-query and attribute-query," International Journal of Computational Intelligence Research, 2007, vol.3, n.1, pp. 6-10.

[10] D. Barbara, et al., "ADAM: a testbed for exploring the use of data mining in intrusion detection," ACM Sigmod Record, 2001, vol. 30, n.4, pp. 15-24.

[11] N. Bhargava, et al., "Decision Tree Analysis on J48 Algorithm for Data Mining," International Journal, 2013. vol.3, n.6.

[12] H. Altwaijry, "Bayesian based intrusion detection system," in IAENG Transactions on Engineering Technologies, 2013, Springer. pp. 29-44.

[13] M. Hall, et al., "The WEKA Data Mining Software: An Update. SIGKDD Explorations,” 2009, vol.11, n.1, pp. 10-18.

[14] M. Anthony., P.L. Bartlett, "Neural Network Learning: Theoretical Foundations" 2009, Campridge University.

[15] S. Rajasekaran, G.A. Pai, "Neural Networks, Fuzzy |Logic and Genetic Algorithm: Synthesis and Applications" 2003, PHI Learning Pvt. Ltd.

[16] D.-z. Wei, Q.-g. Wang, and L.-n. Lin, "Design and Application of a Snort System Based on Data Mining," Journal of Jimei University (Natural Science), 2011, vol.5, pp. 016.

[17] M.A. Hall, "Correlation-based feature selection for machine learning," 2009, The University of Waikato.

[18] J.J. Davis, and A.J. Clark, "Data preprocessing for anomaly based network intrusion detection: A review. computers \& security," 2011, vol.30, n.6, pp. 353-375

[19] P. Kukiełka, Z. Kotulski, (2010) "Adaptation of the neural network-based IDS to new attacks detection", Available from: http://arxiv.org/abs/1009.2406 (Access Date: 17 Oct, 2014).

[20] B. Shanmugam, "Improved Intrusion Detection System Using Fuzzy Logic for Detecting Anamoly and Misuse Type of Attacks" in Proceedings of the Conference of Soft Computing and Pattern Recognition. 2009, pp.212-217. 